\title{
Nicotinamide: Seven new polymorphic structures revealed by melt crystallization and crystal structure prediction
}

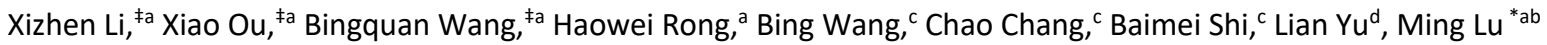 \\ a School of Pharmaceutical Sciences, Sun Yat-sen University, Guangzhou, China \\ ${ }^{b}$ Guangdong Provincial Key Laboratory of New Drug Design and Evaluation, Sun Yat-sen University, Guangzhou, China \\ 'XtalPi Inc., Shenzhen Jingtai Technology Co., Ltd., Shenzhen, China \\ ${ }^{\mathrm{d}}$ School of Pharmacy, University of Wisconsin - Madison, Madison, Wisconsin, USA \\ ${ }^{\ddagger}$ These authors contributed equally.
}

Here, we reported nicotinamide (NIC), a long-known vitamin, was revealed in fact to be a highly polymorphic compound with nine solved single-crystal structures by performing melt crystallization. A CSP calculation successfully identified all six Z' = 1 and 2 experimental structures. Melt crystallization has turned out to be an efficient tool for exploring polymorphic landscape, especially in regions inaccessible by solution crystallization.

Polymorphism has attracted increasing attention from both academic researchers and those in industry ${ }^{1-2}$. Recently, significant progress has been made in the experimental discovery and theoretical prediction of crystal polymorphs. ${ }^{3-4}$ Despite these advances, a key issue in this area is that computational predictions usually yield far more possible polymorphs than are known, raising the famous question posed by Price, "why don't we find more polymorphs?". ${ }^{5}$ It is unclear whether the answer to Price's question lies in the theory (insufficient removal of implausible structures) or experiments (failure to observe certain polymorphs because of slow nucleation, slow growth, instability, or other factors) or both.

Nicotinamide (NIC) is a naturally occurring form of vitamin B3 (Figure 1). Although the study of its polymorphism began in $1943^{6-7}$, the first crystal structure (here denoted $\alpha$ ) was reported in $1954 .{ }^{8}$ In recent decades, NIC has become an extensively used coformer for forming cocrystals with drugs to modify their solubility or other properties. A search in the Cambridge Structural Database (CSD) (version 5.41 updates (Mar 2020)) returned more than 100 structures of organic cocrystals with NIC as a conformer. In the thousands of cocrystal screening experiments performed by various laboratories, only one polymorphic structure was reported in 2011 (here denoted $\beta),{ }^{9}$ and this structure has only been found during an attempt to cocrystallize NIC with the anti-tubercular drug isoxyl.

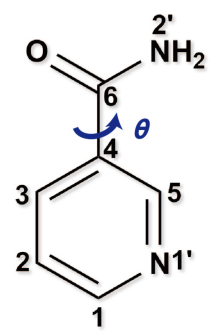

Figure 1. Molecular structure of nicotinamide.

Here, we report seven new polymorphic structures of this well-known vitamin by performing melt crystallization, in addition to two known structures. Single crystals of all nine polymorphs were grown for structural solution by using a novel melt microdroplet strategy. All six experimental structures with one or two molecules in the asymmetric unit $\left(Z^{\prime}=1\right.$ and 2$)$ were found in the lattice energy landscape calculated by crystal structure prediction (CSP). This indicates the potential for melt crystallization to find hidden polymorphs and the prospect of a convergence between experiment and theory.

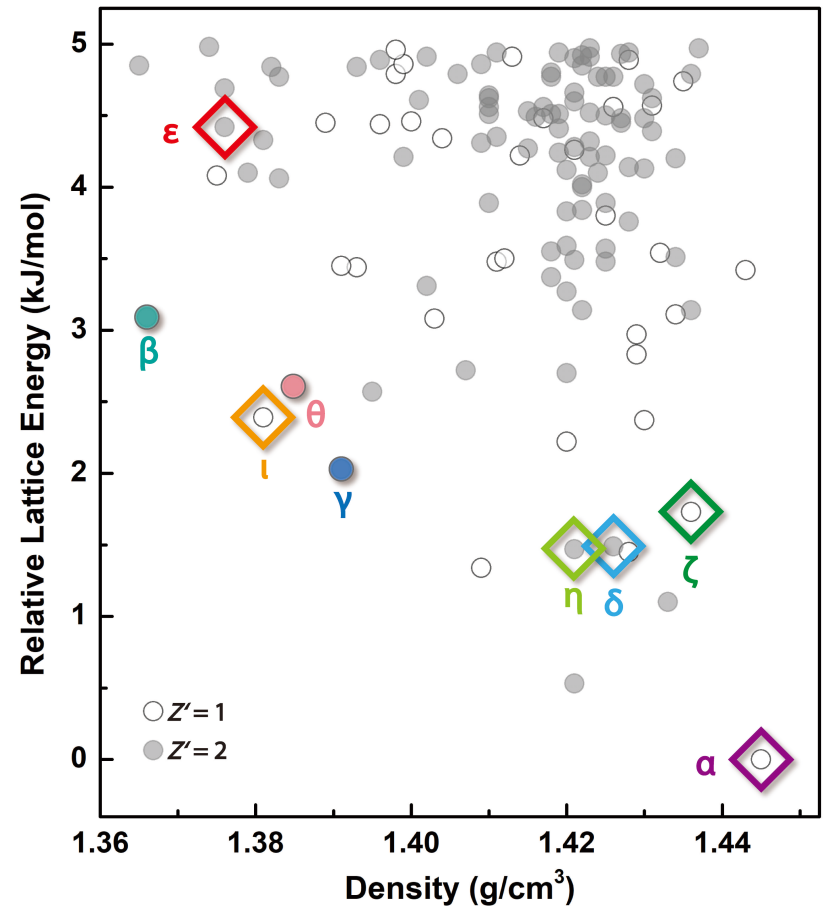

Figure 2. Crystal energy landscape of NIC with $Z^{\prime}=1(0)$ and $Z^{\prime}=2(\bullet)$ All six $Z^{\prime}=1$ and 2 experimental structures were found and are marked with diamond-shaped symbols and the names of the corresponding polymorphs. Two $Z^{\prime}=4$ structures (Forms $\beta$ and $\gamma$ ) and one $Z^{\prime}=20$ structure (Form $\theta$ ) are also marked with dots in this landscape after being optimized and evaluated using the optPBE-vdw functional. 
A CSP calculation of NIC was performed using an in-house cloud platform. ${ }^{10}$ Considering the computational cost, CSPs with $Z^{\prime}=1$ and 2 were performed over the space groups that are most frequently observed in achiral organic crystals (11 space groups for $Z^{\prime}=1$ and 6 space groups for $Z^{\prime}=2$ ). Crystal packing possibilities were surveyed for a range of NIC conformations, defined primarily by the amide-pyridine torsion angle. A system-specific force field was created and applied in the initial generation of possible low-energy structures, and this was followed by further optimization and evaluation using the Perdew-Burke-Ernzerhof functional ${ }^{11}$ with dispersion correction (optPBE-vdw) ${ }^{12}$ implemented in VASP software ${ }^{13-15}$. Figure 1 shows 124 thermodynamically plausible structures within $+5 \mathrm{~kJ} / \mathrm{mol}$ of the global minimum (Figure 2). Form $\alpha$ was identified as the global minimum with an root mean square distance between overlays of 15 molecules $\left(\mathrm{RMSD}_{15}\right)$ of 0.228 $\AA$, and it is close in energy to other structures. This CSP calculation suggests that additional polymorphs might be observed experimentally.

A comprehensive polymorph search was conducted by melt crystallization. Surprisingly, eight polymorphs were obtained from neat NIC melt, including six new Forms: $\gamma, \delta, \varepsilon, \zeta, \eta$ and $\theta$ and the previously known Forms $\alpha$ and $\beta$ according to their powder X-ray diffraction (PXRD) patterns. Form $\alpha$ is the most stable polymorph. It crystallized with a very high growth rate $\left(768 \pm 48 \mu \mathrm{m} / \mathrm{s}\right.$ at $\left.110^{\circ} \mathrm{C}\right)$. Once this form nucleated, it rapidly consumed the remaining liquid and triggered the phase transition of any metastable polymorphs into Form $\alpha$. Of the seven kinetic forms, only four were observed to self-nucleate from the melts: $\delta, \zeta, \eta$ (very rarely) and $\theta$. Forms $\beta$ and $\gamma$ were only obtained as a result of polymorphic transitions from less stable polymorphs, usually from Forms $\theta$ and $\delta$, respectively (Figure 3). Form $\varepsilon$ was observed to only cross-nucleate on the surfaces of Form $\gamma$ and Form $\delta$ above $100{ }^{\circ} \mathrm{C}$. Details of the experimental screen and phase behaviors are described in Supporting information.<smiles>NC(=O)c1ccncc1</smiles>

b)

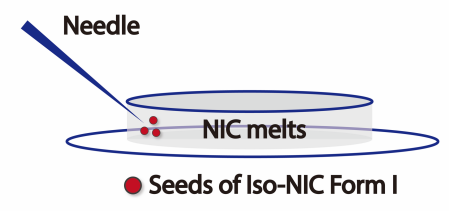

Iso-NIC

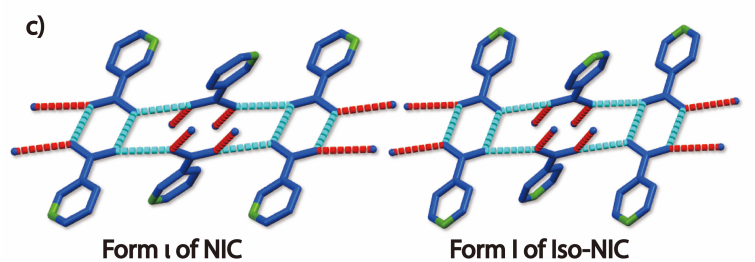

Figure 3. Discovery of (-NIC. a) Molecular structure of iso-NIC. b) Acquisition of L-NIC by seeding iso-NIC Form I in NIC melts from 80$100^{\circ} \mathrm{C}$. c) Hydrogen bonds in NIC Form $\mathrm{t}$ and iso-NIC Form I.

Inspired by the success of pseudo seeding, ${ }^{16-18}$ predicted structures of NIC were compared with six experimental crystal structures of isonicotinamide (iso-NIC), ${ }^{9},{ }^{19-21}$ a structurally similar compound (Figure 3a). This comparison revealed a high similarity between the packing of iso-NIC Form I and that of the "Rank 11" structure of NIC with an RMSD 15 of $0.299 \AA$. Therefore, iso-NIC Form I was seeded into NIC melts at temperatures between 80 and $100{ }^{\circ} \mathrm{C}$, and it successfully triggered the crystallization of another new polymorph (the ninth) of NIC, designated Form I (Figure 3). Introducing iso-NIC seeds into bulk samples placed between two coverslips is much easier to obtain Form I than into surface sample, which usually initial the growth of Form $\alpha$. Form ı has the lowest melting point among nine NIC polymorphs and usually quickly transforms to Form $\alpha$.

Since melt crystallization generally produces crystalline spherulites of insufficient quality for single-crystal X-ray diffraction (SCXRD) investigations, we developed a microdroplet strategy for cultivating single crystals from melt. Following a partial melting of NIC polycrystals, a single-crystal seed was allowed to grow in an isolated microdroplet at 0.98 $0.99 T_{\mathrm{m}}$ (here, $T_{\mathrm{m}}$ refers to the melting point) to avoid the occurrence of noncrystallographic branching and interference from other crystals. Using this method, we successfully grew single crystals of all the NIC polymorphs to a sufficient size and quality for acquiring good SCXRD data and thus determining their single-crystal structures (Figure 4 and Table 1).

a)

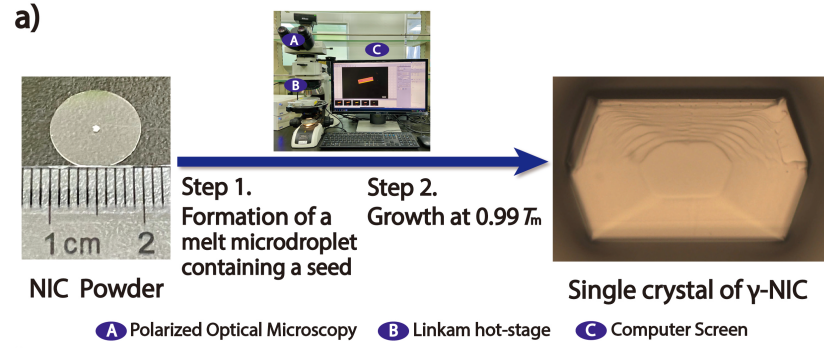

b)
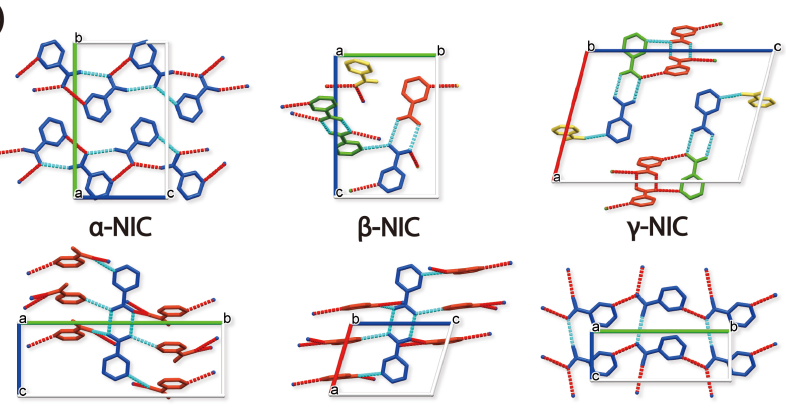

ס-NIC
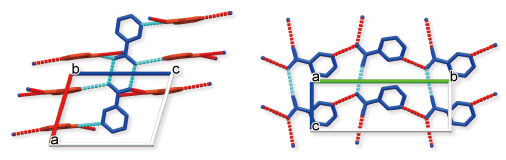

$\varepsilon-\mathrm{NIC}$

క-NIC

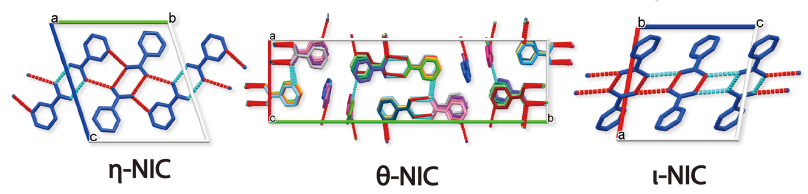

Figure 4. Discovery of NIC polymorphs and determinations of their structures. a) Cultivation of a single crystal of $\gamma$-NIC using hot-stage microscopy. b) Crystal structures of nine NIC polymorphs determined by SCXRD at $100 \mathrm{~K}$ using single crystals grown from melt microdroplets. Forms $\alpha$ and $\beta$ were redetermined for comparison. Colors are used to differentiate crystallographically independent molecules. Forms $\alpha, \beta, \delta$, $\zeta$ and $\eta$ are shown along the a-axis; Forms $\gamma, \varepsilon$ and $\iota$ are shown along the b-axis; Form $\theta$ is shown along the c-axis. 
Table 1. Discovery and structures of nine nicotinamide polymorphs.

\begin{tabular}{|c|c|c|c|c|c|c|c|c|c|}
\hline Polymorphs & Form $\alpha$ & Form $\beta$ & Form $\gamma$ & Form $\delta$ & Form $\varepsilon$ & Form $\zeta$ & Form $\eta$ & Form $\theta$ & Form I \\
\hline Discov. method $^{[a]}[$ ref] & $\mathrm{SC}^{8}$ & $\mathrm{SC}^{9}$ & $\mathrm{MC} / \mathrm{PT}$ & $\mathrm{MC}$ & $\mathrm{MC} / \mathrm{CN}$ & $\mathrm{MC}$ & $\mathrm{MC}$ & $\mathrm{MC}$ & $\mathrm{MC} / \mathrm{CN}$ \\
\hline Yr. str. Solved, & 1954 & 2011 & This work & This work & This work & This work & This work & This work & This work \\
\hline Method [b] [ref] & $\mathrm{SCXD}^{8}$ & $\mathrm{SCXD}^{9}$ & SCXD & SCXD & SCXD & SCXD & SCXD & SCXD & SCXD \\
\hline NICOAM No. [c] & $1^{\text {st }}$ str. & 04 & NA & NA & NA & 07 & 08 & 09 & NA \\
\hline CCDC number [d] & 1866090 & 1866091 & 1866092 & 1984661 & 1984570 & 1893048 & 1893049 & 1893061 & 1984664 \\
\hline$T / \mathrm{K}$ & 100 & 100 & 100 & 100 & 100 & 100 & 100 & 100 & 100 \\
\hline Crystal system & Monoclinic & Monoclinic & Monoclinic & Monoclinic & Triclinic & Monoclinic & Triclinic & Monoclinic & Monoclinic \\
\hline Space group & $\mathrm{P} 2_{1} / \mathrm{c}(14)$ & $\mathrm{P} 2 / \mathrm{n}(13)$ & $\mathrm{P} 2_{1} / \mathrm{c}(14)$ & $\mathrm{P} 2_{1} / \mathrm{c}(14)$ & $P-1(2)$ & $\mathrm{P} 2_{1}(4)$ & $P-1(2)$ & $\mathrm{P} 2_{1}(4)$ & $P 2_{1} / c(14)$ \\
\hline$a / \AA$ & $3.882666(15)$ & 14.9915 (3) & $15.3671(7)$ & $7.3525(3)$ & $7.5564(2)$ & $3.81231(4)$ & $3.7525(3)$ & $10.70050(10)$ & $9.9011(3)$ \\
\hline $\mathrm{b} / \AA$ & $15.6453(5)$ & $10.6814(2)$ & $7.3847(5)$ & 20.7383(9) & $7.9413(2)$ & $14.38794(19)$ & $12.3229(7)$ & $35.1874(2)$ & $5.87732(19)$ \\
\hline$c / \AA$ & $9.3836(3)$ & $15.1888(4)$ & $21.1950(10)$ & $7.4058(4)$ & $10.7974(4)$ & $5.11942(6)$ & $13.0618(6)$ & $15.82560(10)$ & $10.2784(5)$ \\
\hline$\alpha /{ }^{\circ}$ & 90 & 90 & 90 & 90 & $108.100(3)$ & 90 & $71.499(5)$ & 90 & 90 \\
\hline$\beta /{ }^{\circ}$ & $98.394(4)$ & $101.955(2)$ & $104.650(5)$ & 91.044 & $102.596(2)$ & $94.2560(10)$ & $85.676(6)$ & $102.5800(10)$ & 100.003 \\
\hline$\gamma /{ }^{\circ}$ & 90 & 90 & 90 & 90 & $98.293(2)$ & 90 & $85.202(5)$ & 90 & 90 \\
\hline Volume / $\AA^{3}$ & $563.904(4)$ & 2379.43(9) & $2327.0(2)$ & 1129.04 & 585.137 & 280.032 & 570.013 & 5813.37 & 589.028 \\
\hline Z & 4 & 16 & 16 & 8 & 4 & 2 & 4 & 40 & 4 \\
\hline$Z^{\prime}$ & 1 & 4 & 4 & 2 & 2 & 1 & 2 & 20 & 1 \\
\hline$\rho / \mathrm{g} \cdot \mathrm{cm}^{-3}$ & 1.439 & 1.364 & 1.394 & 1.437 & 1.386 & 1.448 & 1.423 & 1.395 & 1.377 \\
\hline R-Factor (\%) & 5.38 & 3.65 & 7. 49 & 6.09 & 5.45 & 2.51 & 6.12 & 6.04 & 6.40 \\
\hline$T_{\mathrm{m}} /{ }^{\circ} \mathrm{C}$ [e] & 129 & 117 & 115 & 114 & 111 & 110.5 & 108 & 105 & 103 \\
\hline Nucleation in $\mathrm{MC}^{[f]}$ & SN & PT & PT & SN & $\mathrm{CN}$ & SN & SN & SN & $\mathrm{CN}$ \\
\hline CSP rank & 1 & Not searched & Not searched & 7 & 92 & 8 & 6 & Not searched & 11 \\
\hline $\mathrm{RMSD}_{15}[\mathrm{~g}] / \AA$ & 0.228 & NA & NA & 0.105 & 0.811 & 0.107 & 0.135 & NA & 0.202 \\
\hline
\end{tabular}

[a] Methods of polymorph discovery: SC, solution crystallization; MC, melt crystallization; PC, polymorphic conversion; $C N$, cross nucleation.

[b] Methods of structural solution: SCXD, single-crystal X-ray diffraction.

[c] NICOAM is the root name for nicotinamide polymorphs in the CSD; NA: NICOAM number is not available at present.

[d] The CCDC numbers and the crystallographic parameters of $\alpha$-NIC and $\beta$-NIC presented in Table 1 are of the single-crystal structures re-determined in this work.

[e] Melting points of nine polymorphs were determined using hot-stage microscopy. The error in $T_{\mathrm{m}}$ is $1{ }^{\circ} \mathrm{C}$ based on at least three measurements.

[f] Nucleation mode in melt crystallization: SN, self-nucleation; PT, polymorphic transformation; CN, cross nucleation.

[g] RMSD 15 is calculated between the experimental structure and the predicted structure using Mercury software; NA: RMSD ${ }_{15}$ values for Forms $\beta, \gamma$ and $\theta$ are not available because these three polymorphs can't be found in CSP landscape due to high Z' $\left(Z^{\prime} \geq 4\right)$. 


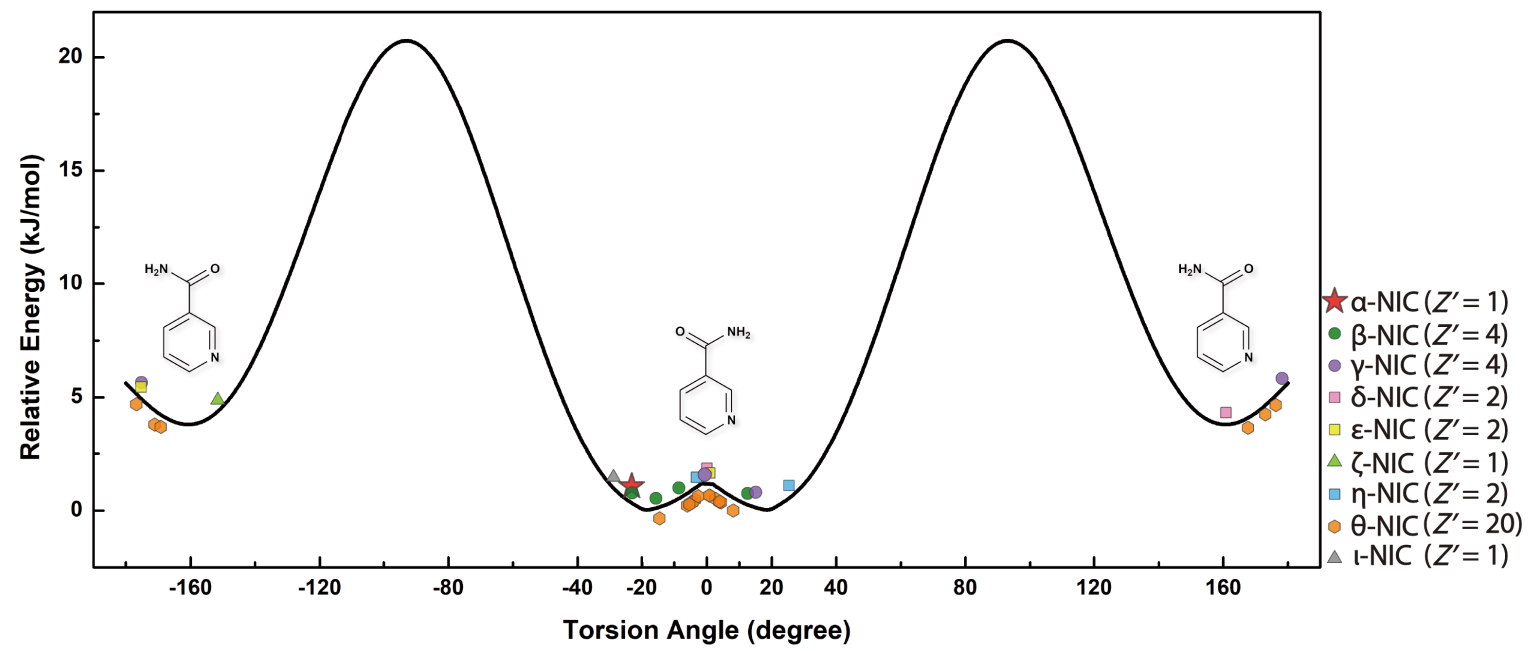

Figure 5. Conformational energy as a function of the torsion angle $\vartheta$ (C5-C4-C6-N2') calculated at the B3LYP/6-31G(d) level using the program GAUSSIAN 09. These calculation results are in good accordance with previously reported data. ${ }^{22,23}$ The experimental conformations found in the nine determined NIC polymorphs are plotted along the two sides of the curve using different colors and symbols.

NIC has two hydrogen bonding acceptors (the amide oxygen and pyridine nitrogen) and two donors (the amide hydrogens), almost all of which are involved in the formation of hydrogen bonds in every polymorph except for Form t. The hydrogen bonding results in amide dimers in all the polymorphs except for Forms $\alpha$ and $\zeta$. Form I shows extremely similar crystal packing and hydrogen bonding with its mother phase, iso-NIC Form I (Figure 3-4). This structural similarity can be fully explained by the fact that the only difference in the chemical structures of the two compounds is the position of the pyridine nitrogen, and this nitrogen atom is not involved in the formation of hydrogen bonds in either structure. Our study also revealed a rare $Z^{\prime}=20$ structure formed in Form $\theta$. In addition to $\theta$-NIC, there are only three other organic compounds having $Z^{\prime} \geq 20$ structures in CSD (version 5.41 updates (Mar 2020)); Refcodes $\operatorname{VUJBAE}\left(Z^{\prime}=20\right)$, OFEREZ $\left(Z^{\prime}=24\right)$, and OGUROZ $\left(Z^{\prime}=56\right)$ ).

The nine polymorphs of NIC have many different conformations with different torsion angles $\vartheta$ (C5-C4-C6-N2'), and all these crystallographic conformations are related to two gas-phase conformers: the global minimum-energy conformer $\left((E)\right.$-Form) with $\vartheta \approx \pm 18^{\circ}(0 \mathrm{~kJ} / \mathrm{mol})$ and the local minimumenergy conformer $((Z)$-Form $))$ with $\vartheta \approx \pm 160^{\circ}(3.6 \mathrm{~kJ} / \mathrm{mol})$ (Figure 5). Therefore, Forms $\alpha / \beta / \eta / \mathrm{l}$ were determined to be related to Forms $\gamma / \delta / \varepsilon / \zeta / \theta$ by conformational polymorphism. ${ }^{2}$ In summary, a variety of conformations, hydrogen bonding modes and crystal packings were found to contribute to the rich polymorphism of NIC.

Successful determination of crystal structures enabled a "blind test" of the CSP calculation. Our CSP identified all three $Z$ ' $=1(\alpha, \zeta, \mathrm{l})$ and all three $Z^{\prime}=2(\delta, \varepsilon, \eta)$ NIC polymorphs (Figure 2). The geometry of the predicted structure is in reasonable agreement with the experimental structure for Form $\varepsilon$ (an $\mathrm{RMSD}_{15}=0.811 \AA$ ); in all other cases, the agreement is excellent $\left(\mathrm{RMSD}_{15}<0.3 \AA\right.$ A). The predicted lattice energy ranking at $0 \mathrm{~K}(\alpha>$ $\eta>\delta>\zeta>\gamma>\iota>\theta>\beta>\varepsilon$ ) has greater discrepancy from the experimental ranking obtained based on the heat of fusion $(\alpha>$ $\beta>\gamma>\zeta)$ or the order of polymorphic transformation $(\alpha>\beta>\gamma>$ $\zeta>\eta>\theta$ ). Such a discrepancy can arise from the neglect of temperature factor and the energy precision of DFT calculations. Nevertheless, the accurate identification of the low-energy crystal structures corresponding to all six experimental polymorphs for the class considered (i.e., $Z^{\prime}=1$ and 2 ) highlights the significant methodological advances that have been achieved in recent years. ${ }^{24,25}$ CSP-generated structures with no experimental analogue motivate further efforts on both computational and experimental sides. Given the nine polymorphs were discovered by melt crystallization at ambient pressure, pressure could be an additional dimension to explore. The result with NIC emphasizes experiments at discovery of hidden polymorphs to more effectively test the accuracy of theoretical models.

Why does melt crystallization produce many more polymorphs of NIC than solution crystallization? In general, the high molecular concentration in a melt results in a much higher thermodynamic driving force for nucleation than that in solution, facilitating the nucleation of metastable polymorphs. ${ }^{26}$ These transient forms could transform into more stable crystal forms and/or cross-nucleate a fast-growing phase, revealing more polymorphs. These metastable phases can be directly observed and then isolated for further structural characterization before phase conversion occurs. All of these factors facilitate the discovery of kinetic polymorphs that are difficult to obtain from solution. Recent work has shown a growing list of polymorphs discovered by melt crystallization: paracetamol ( 3 out of 9), ${ }^{27}$ ROY (4 out of 12), ${ }^{28,29-33}$ griseofulvin ( 2 out of 3$)^{34}$ and vemurafenib ( 3 out of 4 ). ${ }^{35}$ For NIC, 7 out of 9 polymorphs were discovered by melt crystallization. The ability to grow single crystals from melt microdroplets further 
empowers the method, especially to explore the high-energy region of the crystal energy landscape inaccessible using solution crystallization. This advance is important for establishing structure-property relationships using a diverse set of structures, and for understanding the discrepancy between theoretically predicted and experimentally observed crystal structures.

In summary, we have presented our discovery of a nonamorphic system of NIC with nine solved single-crystal structures determined using melt crystallization and CSP. NIC has thus become one of the most structurally well-understood highly polymorphic compounds. Compared with the record holder in this regard (galunisertib ${ }^{6}$ and ROY, 28,33,36 with ten solved structures) and two other compounds with nine solved structures each (aripiprazole ${ }^{21}$ and flufenamic acid ${ }^{37}$ ), NIC is a unique polymorphic system in that the discovery of seven new polymorphic structures was achieved through melt crystallization. We also provided a new example of a computer predicted structure, L-NIC, being found by seeding a templating crystal, iso-NIC Form I. Together with the success in predicting all the observed $Z^{\prime}=1$ and 2 structures of NIC, this work indicates the prospect for improved agreement between theory and experiment. This motivates further progress in both areas, to answer the famously posed question "Why don't we find more polymorphs?".

We are grateful to Yunyun Chen, Prof. Yang Li and Xialin Dai of Sun Yat-sen University and Kunlin Wang of the University of Minnesota for their helpful discussions regarding the structural solutions. We thank Dr. Guangxu Sun, Dr. Shigang Ruan and Sizhu Li of XtalPi Inc. for their beneficial discussions in the CSP research. We are grateful for the financial support of this work from the Guangdong Basic and Applied Basic Research Foundation (No. 2020A151501782), the Natural Science Foundation of Guangdong Province (No. 2018A030313335 and No. 2016A030312013) and the Guangdong Provincial Key Laboratory of Construction Foundation (No. 2017B030314030).

\section{Conflicts of interest}

The authors declare no conflicts of interest.

\section{References}

1 A. J. Cruz-Cabeza, S. M. Reutzel-Edens and J. Bernstein, Chemical Society Reviews, 2015, 44, 8619-8635.

2 O. A. Rahal, C. E. Hughes, P. A. Williams, A. J. Logsdail, Y. Diskin-Posner and K. D. M. Harris, Angewandte ChemieInternational Edition, 2019, 58, 18788-18792.

3 S. L. Price, Proceeding Royal Society A, 2018, 474

4 R. M. Bhardwaj, J. A. McMahon, J. Nyman, L. S. Price, S. Konar, I. D. H. Oswald, C. R. Pulham, S. L. Proce and S. M. ReutzelEdens, Journal of the American Chemistry Society, 2019, 141, 13887-13897.

5 S. L. Price, Acta Crystallographica Section B-Structural Science Crystal Engineering and Materials, 2013, 69, 313-328.

6 L. Kofler and A. Kofler. Berichte der Deutschen chemischen Gesellschaft. 1943, 76, 246-248.
7 T. Hino, J. L. Ford, M. W. Powell, Thermochim Acta, 2001, 374, 85-92.

8 W. B. Wright and G. S. D. King, Acta Crystallographica, 1954 7, 283-288

9 J. J. Li, S. A. Bourne and M. R. Caira, Chemical Communications, 2011, 47, 1530-1532.

10 P. Y. Zhang, G. P. F. Wood, J. Ma, M. J. Yang, Y. Liu, G. X. Sun, Y. A. Jiang, B. C. Hancock and S. H. Wen, Crystal Growth \& Design, 2018, 18, 6891-6900.

11 J. P. Perdew, K. Burke and M. Ernzerhof, Physical Review Letters, 1996, 77, 3865.

12 J. Klimeš, D. R. Bowler and A. Michaelides, Physical Review B, 2011, 83, 195131.

13 G. Kresse and J. Furthmüller, Computational Materials Science, 1996, 6, 15-50.

14 G. Kresse and J. Hafner, Physical Review B, 1993, 47, 558.

15 G. Kresse and J. Hafner, Physical Review B, 1994, 49, 14251.

16 J. B. Arlin, L. S. Price, S. L. Price and A. J. Florence, Chemical Communications, 2011, 47, 7074-7076.

17 D. K. Bucar, G. M. Day, I. Halasz, G. G. Z. Zhang, J. R. G. Sander, D. G. Reid, L. R. MacGillivray, M. J. Duer and W. Jones, Chemical Science, 2013, 4, 4417-4425.

18 T. A. Zeidan, J. T. Trotta, P. A. Tilak, M. A. Oliveira, R. A. Chiarella, B. M. Foxman, O. Almarsson and M. B. Hickey, CrystEngComm, 2016, 18, 1486-1488.

19 A. I. Vicatos and M. R. Caira, CrystEngComm, 2019, 21, 843 849.

20 K. S. Eccles, R. E. Deasy, L. Fabian, D. E. Braun, A. R. Maguire and S. E. Lawrence, CrystEngComm, 2011, 13, 6923-6925.

21 C. B. Aakeroy, A. M. Beatty, B. A. Helfrich and M. Nieuwenhuyzen, Crystal Growth \& Design, 2003, 3, 159-165.

22 Y. Miwa, T. Mizuno, K. Tsuchida, T. Taga and Y. Iwata, Acta Crystallographica Section B, 1999, 55, 78-84.

23 B. Vogelsanger, R. D. Brown, P. D.Godfrey and A. P. Pierlot, Journal of Molecular Spectroscopy, 1991, 145, 1-11.

24 H. C. S. Chan, J. Kendrick and F. J. J. Leusen, Angewandte Chemie International Edition, 2011, 50, 2979-2981.

25 S. Roy and A. J. Matzger, Angewandte Chemie International Edition, 2009, 48, 8505-8508.

26 V. M. Fokin, E. D. Zanotto, N. S. Yuritsyn and J. W. P. Schmelzer, Journal of Non-Crystalline Solids, 2006, 352, 26812714.

27 C. T. Hu, A. G. Shtukenberg, M. Tan, L. Vogt-Maranto, E. J. Chan, W. Q. Xu, J. X. Yang, M. E. Tuckerman and B. Kahr, Acta Crystallographica Section A, 2019, 75, 328.

28 K. S. Gushurst, J. Nyman and S. X. M. Boerrigter, CrystEngComm, 2019, 21, 1363-1368.

29 L. Yu, Accounts of Chemical Research, 2010, 43, 1257-1266.

30 S. Chen, I. A. Guzei and L. Yu, Journal of the American Chemical Society, 2005, 127, 9881-988.

31 S. Chen, H. M. Xi and L. Yu, Journal of the American Chemical Society, 2005, 127, 17439-17444.

32 L. Yu, G. A. Stephenson, C. A. Mitchell, C. A. Bunnell, S. V. Snorek, J. J. Bowyer, T. B. Borchardt, J. G. Stowell and S. R. Byrn, Journal of the American Chemical Society, 2000, 122, 585-591.

33 A. R. Tyler, R. Ragbirsingh, C. J. McMonagle, P. G. Waddell, S. E. Heaps, J. W. Steed, P. Thaw, M. J. Hall and M. R. Probert, Chem, 2020, 6, 1-11.

34 A. Mahieu, J. F. Willart, E. Dudognon, M. D. Eddleston, W. Jones, F. Danede and M. Descamps, Journal of Pharmaceutical Sciences, 2013, 102, 462-468.

35 M. Lu and L. S. Taylor, Crystal Growth \& Design, 2016, 16, 6033-6042.

36 J. Nyman, L. Yu and S. M. Reutzel-Edens, CrystEngComm, 2019, 21, 2080-2088.

37 V. Lopez-Mejias, J. W. Kampf and A. J. Matzger, Journal of the American Chemical Society, 2012, 134, 9872-9875. 